\title{
The influence of rest period instructions on the default mode network
}

\section{Christopher Benjamin ${ }^{1,2+}$, Daniel A. Lieberman ${ }^{2+}$, Maria Chang ${ }^{1}$, Noa Ofen $^{3}$, Sue Whitfield-Gabrieli ${ }^{3}$, John D. E. Gabrieli ${ }^{3}$ and Nadine Gaab ${ }^{1,2 *}$}

\author{
'Laboratories of Cognitive Neuroscience, Developmental Medicine Center, Children's Hospital Boston, Boston, MA, USA \\ 2 Harvard Medical School, Boston, MA, USA \\ ${ }^{3}$ Department of Brain and Cognitive Sciences, Massachusetts Institute of Technology, Cambridge, MA, USA
}

\section{Edited by:}

Kenneth Hugdahl, University of

Bergen, Norway

Reviewed by:

Tom Eichele, University of Bergen, Norway

Martin Walter, Otto-Von-GuerickeUniversität Magdeburg, Germany

${ }^{*}$ Correspondence:

Nadine Gaab, Laboratories of Cognitive Neuroscience, Developmental

Medicine Center, Children's Hospital

Boston, Harvard Medical School,

1 Autumn Street, Mailbox \#713,

Boston, MA 02215, USA.

e-mail:nadine.gaab@childrens.harvard. edu

${ }^{+}$Christopher Benjamin and

Daniel A. Lieberman have contributed equally to this work.
The default mode network (DMN) refers to regional brain activity that is greater during rest periods than during attention-demanding tasks; many studies have reported DMN alterations in patient populations. It has also been shown that the DMN is suppressed by scanner background noise (SBN), which is the noise produced by functional magnetic resonance imaging (fMRI). However, it is unclear whether different approaches to "rest" in the noisy MR environment can alter the DMN and constitute a confound in studies investigating the DMN in particular patient populations (e.g., individuals with schizophrenia, Alzheimer's disease). We examined 27 healthy adult volunteers who completed an $\mathrm{fMRI}$ experiment with three different instructions for rest: (1) relax and be still, (2) attend to SBN, or (3) ignore SBN. Region of interest analyses were performed to determine the influence of rest period instructions on core regions of the DMN and DMN regions previously reported to be altered in patients with or at risk for Alzheimer's disease or schizophrenia. The dorsal medial prefrontal cortex (dmPFC) exhibited greater activity when specific resting instructions were given (i.e., attend to or ignore SBN) compared to when non-specific resting instructions were given. Condition-related differences in connectivity were also observed between regions of the dmPFC and inferior parietal/posterior superior temporal cortex. We conclude that rest period instructions and SBN levels should be carefully considered for $\mathrm{fMRI}$ studies on the DMN, especially studies on clinical populations and groups that may have different approaches to rest, such as first-time research participants and children.

Keywords: default mode, fMRI, resting state, scanner background noise

\section{INTRODUCTION}

Functional neuroimaging studies have identified a network of brain regions associated with the absence of a specific task or action that supports ongoing, internal modes of cognition (Raichle and Snyder, 2007; Buckner et al., 2008). This network has been termed the "default mode of brain function" or "default mode network" (DMN) because it is consistently observed in the absence of goaldirected behaviors or attention-demanding stimuli (Raichle et al., 2001; Greicius et al., 2003; Buckner et al., 2008). The DMN consists of several regions, including ventral medial prefrontal cortex, dorsal medial prefrontal cortex (dmPFC), inferior parietal cortex, lateral temporal cortex, posterior cingulate, and parahippocampal regions (reviewed in Buckner et al., 2008).

Default mode network activation has been observed in studies that vary by neuroimaging technique and cognitive task. Studies using positron emission tomography (PET; Shulman et al., 1997; Raichle et al., 2001) and functional magnetic resonance imaging (fMRI; Binder et al., 1999; Greicius et al., 2003) have found greater activity in the DMN during rest conditions as compared to active task conditions. These findings do not depend on a particular cognitive task; the DMN has been found to deactivate during visual tasks (e.g., Shulman et al., 1997; Mazoyer et al., 2001), auditory tasks (e.g., Mazoyer et al., 2001), language tasks (e.g., Mazoyer et al., 2001), and working memory tasks (e.g., Fransson, 2006).
It has been hypothesized that DMN activity represents a functional baseline for human brain activity (Raichle et al., 2001). More specifically, it has been suggested that the DMN supports spontaneous stimulus-independent thoughts or "mind wandering" (Mason et al., 2007). Several studies suggest that these thoughts consist of memories and past experiences that are used to solve problems, plan for future actions, or create general self-relevant mental simulations (Binder et al., 1999; Mazoyer et al., 2001; Buckner et al., 2008). Medial prefrontal regions in particular have been implicated in supporting self-referential mental activity and integrating ongoing emotional processes into decision-making (Gusnard and Raichle, 2001; Gusnard et al., 2001). The DMN has also been hypothesized to automatically monitor the external environment for salient events, such as novel or unexpected movement patterns or socially relevant stimuli (Gusnard and Raichle, 2001). The precise functions of the DMN are still an active topic of investigation, with recent evidence demonstrating DMN activation during unconscious states, such as sleep or anesthesia (Gusnard and Raichle, 2001; Fox and Raichle, 2007).

The hypothesized functions of the DMN are necessary for typical cognition, and some DMN regions are compromised by neurological and psychiatric disease (Buckner et al., 2008; Broyd et al., 2009). Several studies have shown decreased activity in and connectivity between DMN structures in patients with Alzheimer's disease and 
individuals at risk for developing Alzheimer's disease (Lustig et al., 2003; Greicius et al., 2004; Rombouts et al., 2005; Sauer et al., 2006; Wang et al., 2006; Firbank et al., 2007; He et al., 2007, Liu et al., 2008). These findings have led to the hypothesis that Alzheimer's disease is associated with an overall deficit in baseline neural activity during the resting state, which consequently affects task-related activations/deactivations and hinders cognitive performance (Wermke et al., 2008). Similarly, hyperconnectivity and hyperactivity of DMN components have been observed in schizophrenia (Garrity et al., 2007; Zhou et al., 2007; Whitfield-Gabrieli et al., 2009). In some studies, DMN alterations were directly correlated with schizophrenic symptomatology (Bluhm et al., 2007; Garrity et al., 2007; Harrison et al., 2007; Pomarol-Clotet et al., 2008; Whitfield-Gabrieli et al., 2009). These differences in DMN activity have been interpreted as hypersensitivity to internal thoughts and the external environment (Broyd et al., 2009); consequently, individuals with schizophrenia may mismanage attentional resources at the cost of cognitive performance (Whitfield-Gabrieli et al., 2009). More recent studies report atypical DMN activation in a variety of conditions including depression, attention deficit hyperactivity disorder, and autism spectrum disorders (Kennedy et al., 2006; Tian et al., 2006; Greicius et al., 2007; Kennedy and Courchesne, 2008).

Though underlying differences in neural function likely underlie some of the observed differences between DMN activity of patients and healthy controls, it has been proposed that differences in the ability to rest within the MR environment itself may also contribute to these differences (see discussion in, e.g., Morcom and Fletcher, 2007; Raichle and Snyder, 2007). McAvoy et al. (2008) showed that changes in the way individuals rest in terms of their eye position (eyes open, eyes closed or eyes open with fixation) were associated with changes within the DMN network.

Furthermore, it has been shown that scanner background noise (SBN) accompanying the resting state can also alter the DMN (Gaab et al., 2008). Unlike PET, fMRI produces SBN, which can reach levels of up to $130 \mathrm{~dB}$ specific pressure level (SPL). Depending on the characteristics of the scanner and the pulse sequence used, this is comparable to the noise produced by a nearby jackhammer (Ravicz et al., 2000; Price et al., 2001; Amaro et al., 2002), though headphones and/or earplugs attenuate this somewhat. There is EEG evidence that some ERP components are altered by MR scanner noise (e.g., Novitski et al., 2001). In auditory cortex as measured by $\mathrm{fMRI}, \mathrm{SBN}$ induces BOLD responses during control conditions, potentially masking task-related auditory activations (Talavage and Edmister, 2004; Gaab et al., 2007). Additionally, the effect of SBN on auditory regions may not be consistent across conditions; therefore, contrasting two conditions with SBN does not necessarily result in SBN-induced BOLD responses canceling out (Talavage and Edmister, 2004; Tomasi et al., 2005; Gaab et al., 2007). More importantly, Gaab et al. (2008) examined DMN activity during silent rest intervals without SBN versus rest intervals with SBN and found that DMN activity during rest was suppressed in the presence of SBN.

Under non-specific instructions such as "relax" or "stay still and do nothing," some participants may actively attend to SBN while others may ignore SBN. Different approaches may alter attentional demands and DMN activity; differences in how groups treat rest are particularly likely to occur in certain patient populations, where in spite of clear instructions a group's pathology may influence their resting approach, confounding observed group differences. Furthermore, given the substantial evidence of an aberrant DMN in Alzheimer's disease and schizophrenia (Lustig et al., 2003; Greicius et al., 2004; Rombouts et al., 2005; Celone et al., 2006; Wang et al., 2006; Bluhm et al., 2007; Firbank et al., 2007; Garrity et al., 2007; Harrison et al., 2007; He et al., 2007; Sorg et al., 2007; Zhou et al., 2007; Bai et al., 2008; Liu et al., 2008; Persson et al., 2008; Whitfield-Gabrieli et al., 2009), it is possible that some of those observed differences may be explained by different approaches to SBN during rest periods in patient populations. For example, it may be possible that patients with auditory hallucinations attend to the SBN differently than their healthy control group, which could lead to a systematic bias.

The goal of this study was to determine whether different approaches to SBN during a rest condition affect parts of the DMN network and therefore could potentially bias observed activation in DMN components when comparing groups. We scanned healthy volunteers under different rest conditions, during which they were asked to either "relax and stay still," or actively "attend to" or "ignore" SBN. We compared activations during these three "rest" conditions in regions of interest (ROI) defined by anatomical areas of the DMN in a healthy population ("typical" ROIs) and a set of DMN regions reported to be altered in patients with or at risk for Alzheimer's disease or patients with schizophrenia ("clinical" ROIs). We used the "clinical" ROIs to determine if the possible effect of rest period instructions might alter activation in regions of the DMN and interpretation of study results in clinical populations. Additionally, we performed connectivity analyses for the key regions of the DMN for each condition using both the "typical" and "clinical" ROI regions as seeds. Further understanding of this methodological issue may highlight the importance of ensuring patient populations approach "rest" conditions in a uniform way and promote MR protocols in which SBN is minimized.

\section{MATERIALS AND METHODS PARTICIPANTS}

Twenty-seven healthy adult volunteers (12 females, age 18-42, mean $=28.9)$ participated in this study. Participants gave written consent in accordance with the institutional review board at MIT.

\section{EXPERIMENTAL PROCEDURE}

The experimental paradigm was divided into two phases (Figure 1). The first phase was designed to measure DMN activity under a non-specific resting instruction (baseline). During baseline, participants were instructed to relax and lie still in the scanner and to fixate on the displayed fixation cross. During this phase, subjects were given no instructions with regard to SBN. For the second phase of the experiment (specific instruction phase), participants were given specific instructions with regard to SBN; participants were instructed to alternate between attending to and ignoring SBN while fixating on the displayed cross. A block design with two conditions and eight blocks per condition was used for the specific instruction phase. Before each block, a 2-s visual cue instructed participants to actively attend to or ignore SBN. Cues were followed by a 20 -s block in which a fixation cross was presented. The specific instruction phase was designed to measure DMN activity 


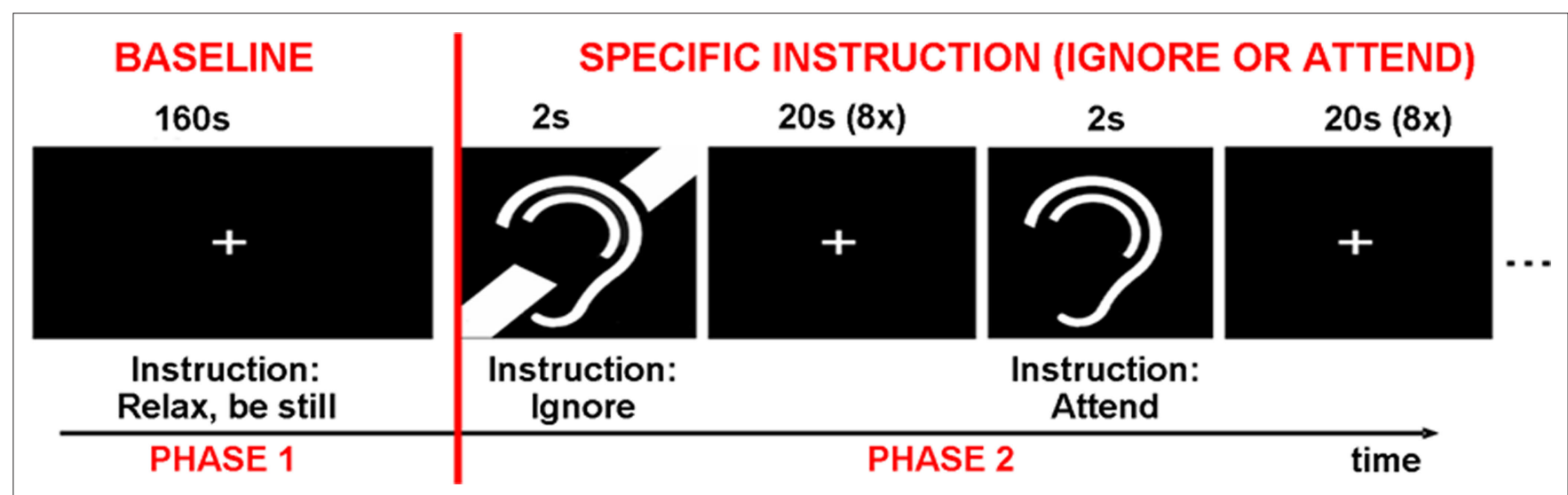

FIGURE 1 |The experiment was divided into two phases. During the baseline phase, participants were instructed to relax and be still. During the specific instruction phase, participants were presented with alternating cues for attend to or ignore scanner background noise.

while subjects attended to or ignored SBN. It is important to note that all subjects received the specific instructions (when to actively ignore or attend SBN) after the initial baseline phase, and therefore we think that the initial baseline phase represents a resting baseline as implemented in many studies which aim to assess the DMN. Each of the three conditions (baseline, attend, ignore) had a total duration of $160 \mathrm{~s}$. The total duration of scan time was approximately $8.5 \mathrm{~min}$.

\section{IMAGING PROCEDURE}

Functional MR images were collected at the Athinoula A. Martinos Imaging Center at the McGovern Institute for Brain Research, MIT. The images were acquired using a 3.0-Tesla Tim Trio Siemens scanner using a Matrix receive head coil and a whole-body transmit coil. BOLD measures were collected using single-shot gradient echo sequences covering the entire brain ( 33 slices, $\mathrm{TR}=2000 \mathrm{~ms}$, $\mathrm{TE}=30 \mathrm{~ms}, \mathrm{FOV}=200 \mathrm{~mm} \times 200 \mathrm{~mm}$, matrix $64 \times 64$, flip angle $90^{\circ}$, in plane resolution $3.1 \mathrm{~mm} \times 3.1 \mathrm{~mm}, 5 \mathrm{~mm}$ slice thickness with $1 \mathrm{~mm}$ slice skip). Separate runs were used for the two phases (baseline and specific instruction) of the experiment. Eighty brain images were acquired for each condition (baseline, attend, ignore).

\section{MR IMAGE ANALYSIS}

All images were preprocessed using SPM $2^{1}$. After reconstruction, images were realigned to the first image in the sequence, spatially normalized to SPM2's echo planar image (EPI) template and smoothed with a 4-mm isotropic Gaussian kernel.

Outliers were identified using the Brain Imaging Toolbox ${ }^{2}$ on the basis of mean signal intensity and head movement relative to the previous scan. Images with mean signal intensity three standard deviations or more beyond the mean were excluded from statistical analysis. Images with head movement exceeding $0.5 \mathrm{~mm}$ or 0.015 radians for translational and rotational movement respectively (relative to the previous scan) were also excluded from further analysis. On average, 2.6 scans were excluded per subject.

${ }^{1}$ http://www.fil.ion.ucl.ac.uk/spm/

${ }^{2}$ http://web.mit.edu/swg/software.htm
We adopted a similar approach to a number of studies observing group differences in DMN activation (e.g., Rombouts et al., 2005; Pomarol-Clotet et al., 2008) and statistically analyzed our data using the general linear model (GLM; as implemented in SPM2; Friston et al., 1995). Each of the three conditions (baseline, attend, ignore) were convolved with the hemodynamic response function. Block regressors were used for each run in the first-level analysis to remove any baseline signal differences between the two runs. The following contrasts were calculated for each subject: [attend $>$ baseline], [ignore $>$ baseline], and [ignore $>$ attend].

As a quality assurance check, we sought to exclude participants who did not shift their attention toward SBN (between attending and ignoring) during the specific instruction phase of the experiment. However, we could not check against any behavioral measures because they were not collected. Consequently, correct completion of the specific instruction phase was estimated by ROI analyses within bilateral Heschl's gyri (defined by the AAL atlas as implemented in WFU PickAtlas ${ }^{3}$ ). It has been shown that auditory attention modulates activity in primary auditory cortex (e.g., Jancke et al., 1999). Thus, participants who attended to and ignored SBN according to the visual cues should exhibit greater primary auditory cortex activation in the [attend > ignore] contrasts (Figure 2). Only participants with positive weighted parameter estimates in bilateral Heschl's gyri in the [attend > ignore] contrast were included for further analysis $(n=17$, seven females, age 18-37, mean $=28.1$ ). Three of the excluded participants reported that they fell asleep during the task and one of the excluded participants reported that she mixed up the instruction cues.

\section{REGION OF INTEREST ANALYSES}

In order to objectively determine if any DMN regions were influenced by different instructions for the rest period, ROI analyses were conducted by extracting mean weighted parameter estimates from the contrasts listed above for each subject using anatomical landmarks for DMN ROIs identified by Buckner et al. (2008). Anatomical landmarks were defined by the AAL atlas as implemented in WFU pick $\operatorname{atlas}^{4}$ (Tzourio-Mazoyer et al., 2002; Maldjian et al., 2003, 2004).

${ }^{3}$ http://fmri.wfubmc.edu/cms/software ${ }^{4}$ http://fmri.wfubmc.edu/cms/software 


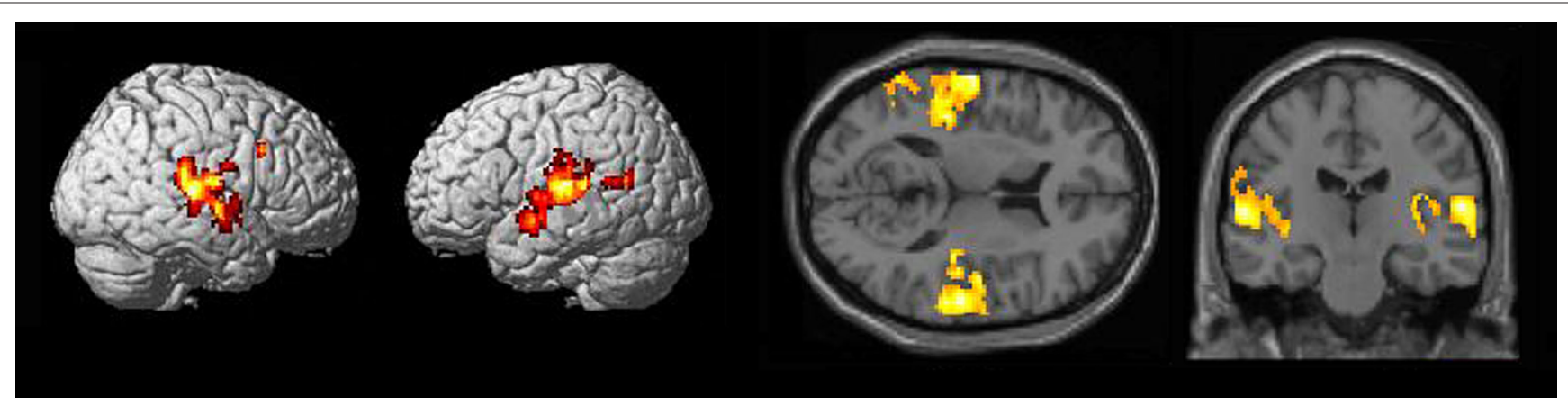

FIGURE 2 | Surface rendering and section views of auditory activation in [attend SBN $>$ ignore SBN] contrasts $(n=17, p<0.005$ uncorrected).

These "typical" ROIs were defined for the six core regions of the DMN (as reviewed in Buckner et al., 2008): (1) ventral medial prefrontal cortex, (2) dmPFC, (3) hippocampal formation, (4) lateral temporal cortex, (5) posterior cingulate, and (6) inferior parietal lobule. Since these ROIs were defined using the AAL atlas, they are based on the high-resolution single-subject T1 image provided by MNI. Extracted weighted parameter estimates (for each of the three contrasts described above) in typical ROIs were entered into a one-sample $t$-test (test value $=0$ ) with Bonferroni correction for multiple comparisons $(p<0.008)$.

The second goal of this study was to examine how DMN regions that may be influenced by different approaches to rest correspond with those regions reported to be altered in DMN studies on patients with Alzheimer's disease or schizophrenia.

To answer this question we first conducted a second set of ROI analyses using "clinical" ROIs based on previous studies examining the DMN in patients with or at risk for Alzheimer's disease and in patients with schizophrenia. Clinical ROIs were defined as 3D spheres (radius $=10 \mathrm{~mm}$ ) centered on independently reported coordinates of peak DMN differences between healthy controls and patients with schizophrenia, patients with Alzheimer's disease and individuals at risk for developing Alzheimer's disease. Unlike the "typical" ROIs, which were based on the MNI T1 brain, clinical ROIs were based on coordinates reported in the DMN literature. To obtain these coordinates, we referred to all available published manuscripts that investigated the DMN in patients with schizophrenia, patients with Alzheimer's disease, and individuals at risk for developing Alzheimer's disease. Only research articles that reported Talairach or MNI coordinates were used for creating clinical ROIs (Lustig et al., 2003; Greicius et al., 2004; Rombouts et al., 2005; Celone et al., 2006; Sauer et al., 2006; Wang et al., 2006; Bluhm et al., 2007; Garrity et al., 2007; Harrison et al., 2007; He et al., 2007; Sorg et al., 2007; Zhou et al., 2007; Bai et al., 2008; Persson et al., 2008; Pomarol-Clotet et al., 2008; Whitfield-Gabrieli et al., 2009). Only clinical ROIs within dmPFC were examined because this was the only region in which activation between rest periods with different instructions significantly differed in our study. Clinical ROIs were considered within dmPFC if they had at least $50 \%$ overlap with the anatomically defined "typical" dmPFC ROI. Five studies reported a total of eight ROIs (see Table 1) within dmPFC meeting these criteria (Greicius et al., 2004; Garrity et al., 2007; Sorg et al., 2007; Zhou et al., 2007; Persson et al., 2008). For each clinical ROI within
Table 1 | Clinical DMN regions of interest within dorsal medial prefrontal cortex.

\begin{tabular}{llrrr}
\hline Paper & Disorder & $\boldsymbol{x}$ & $\boldsymbol{y}$ & $\boldsymbol{z}$ \\
\hline Garrity et al. (2007) & Schizophrenia & 9 & 52 & 5 \\
Garrity et al. (2007) & Schizophrenia & -3 & 45 & 24 \\
Garrity et al. (2007) & Schizophrenia & 9 & 54 & 24 \\
Greicius et al. (2004) & Alzheimer's disease & 8 & 64 & 4 \\
Persson et al. (2008) & (at risk for) Alzheimer's disease & -4 & 59 & 16 \\
Sorg et al. (2007) & (at risk for) Alzheimer's disease & 6 & 63 & 27 \\
Zhou et al. (2007) & Schizophrenia & -3 & 54 & 6 \\
Zhou et al. (2007) & Schizophrenia & 3 & 51 & 21 \\
& & & & \\
\hline
\end{tabular}

Coordinates are reported in MNI space. Coordinates that were reported in Talairach space in the original publication were converted to MNI space using Matthew Brett's non-linear transformation (http://imaging.mrc-cbu.cam.ac.uk/ imaging/MniTalairach).

dmPFC, weighted parameter estimates were extracted from each contrast and entered into a one-sample $t$-test (test value $=0)$ with Bonferroni correction for multiple comparisons $(p<0.006)$.

Secondly, having examined rest instruction-related changes in activation within DMN structures (defined structurally and with reference to the clinical literature), we examined whether instructions could similarly alter the connectivity between $\mathrm{DMN}$ regions. We first performed connectivity analyses for each of the three conditions using the key regions of the DMN as seeds (Buckner et al., 2008). We then completed secondary connectivity analyses using the $\mathrm{AD}$ and schizophrenia $\mathrm{ROI}$ from the previous analysis that demonstrated the greatest betweencondition differences as seeds (see result section below). Analyses were completed using the conn toolbox (v0.1 Castanon, 20095). Preprocessing of participants' structural images included normalization and segmentation. Functional images were smoothed, normalized to MNI-152 space, and temporally filtered $(0 \mathrm{~Hz}<\mathrm{f}<0.25 \mathrm{~Hz})$. Confounding signal related to white matter and CSF (five dimensions) as well as movement (six dimensions with first order derivatives) was considered separately. Sessions were analyzed individually. Conditions were weighted with a hanning window to reduce block on/offset effects. $\mathrm{R}$ maps of the bivariate correlation between each averaged (structural) ROI time-course and all other voxels were created. These values were

${ }^{5}$ http://web.mit.edu/swg/software.htm 
Fisher transformed and contrasted at the group-level (SPM8). The false discovery rate was used to account for multiple comparisons, with a corrected significance level of $p<0.05$ (Benjamini and Hochberg, 1995; Benjamini and Yekutieli, 2001).

\section{RESULTS}

Typical DMN ROI analyses determined that activation only in dmPFC was influenced by instructions regarding SBN during rest periods (Figure 3). Weighted parameter estimates were significantly greater in $\mathrm{dmPFC}$ when participants were instructed to attend to SBN compared to the baseline phase ([attend $>$ baseline], $t(16)=4.19$, $p<0.001)$. Similarly, greater activation was observed in dmPFC when participants were instructed to ignore SBN compared to the baseline phase ([ignore $>$ baseline], $t(16)=5.88, p<0.000$ ). There was no significant difference between attending to SBN versus ignoring SBN in dmPFC ([ignore $>$ attend], $t(16)=0.905 p=0.379$ ). There were no significant differences among the three conditions for any of the other typical ROIs (ventral medial prefrontal cortex, posterior cingulate, lateral temporal cortex, inferior parietal lobule, hippocampal formation, all: $p>0.068$ ). These results show increased activation only in $\mathrm{dmPFC}$ when participants were instructed to attend to or ignore SBN compared to the baseline phase.

Only clinical ROIs with at least $50 \%$ overlap with significant typical ROIs were used for further analysis (Figure 4). A total of eight clinical ROIs had at least 50\% overlap with dmPFC (see Table 1). Of those eight, five had significantly greater activation during the ignore SBN condition compared to the baseline condition ([ignore $>$ baseline], $p<0.001$ ). These five regions include medial and superior frontal cortex regions found in previous studies by Garrity et al. (2007), Zhou et al. (2007), and Persson et al. (2008). Two clinical ROIs were significantly greater during the attend SBN condition compared to the baseline condition ([attend > baseline], $p<0.001)$. These two regions include medial frontal regions from Garrity et al. (2007) and Zhou et al. (2007) (Figure 4). There were no significant differences between attend and ignore conditions for any of the eight clinical ROIs ([attend > ignore], all: $p>0.146$ ). This analysis of clinical ROIs shows that regions altered by attend/ ignore SBN instructions (i.e., dmPFC) coincide with DMN regions observed in previous studies to differ in activation between patient populations and typical individuals.

Finally, these results were extended through a functional connectivity analysis. Initial analyses using the above structurally defined ROI as seed regions revealed expected positive correlations between regions of the DMN including the DMPFC, inferior parietal, lateral temporal, and posterior and anterior cingulate regions (Figure 5). We then examined whether changes in rest instruction altered connectivity from regions of the dmPFC implicated in studies of the DMN in clinical populations. This was achieved using the clinical ROI that had demonstrated the most marked between-condition differences in the initial analyses; one ROI from both the schizophrenia (Zhou et al., 2007) and AD (Persson et al., 2008) literature were used as functional connectivity seeds.

When the dmPFC ROI derived from Zhou et al. (2007) was examined, increases in dmPFC-parietal connectivity were observed in instructed conditions as compared to baseline (Figure 6). In the contrast [ignore $>$ baseline], the left inferior parietal lobule $[t(16)=5.13 ; p<0.01]$ correlated significantly more with dmPFC activity during the ignore condition. When the "attend" condition was directly compared with the baseline, connectivity was significantly greater in both the left $[t(16)=5.05 ; p<0.01]$ and right $[t(16)=4.79 ; p<0.01]$ inferior parietal regions. Additional clusters fell within dorsal frontal regions, including right middle frontal $[t(16)=5.14 ; p<0.05]$ and left superior frontal $[t(16)=5.1$; $p<0.05]$ gyri and the left cuneus $[t(16)=4.37, p<0.05]$.

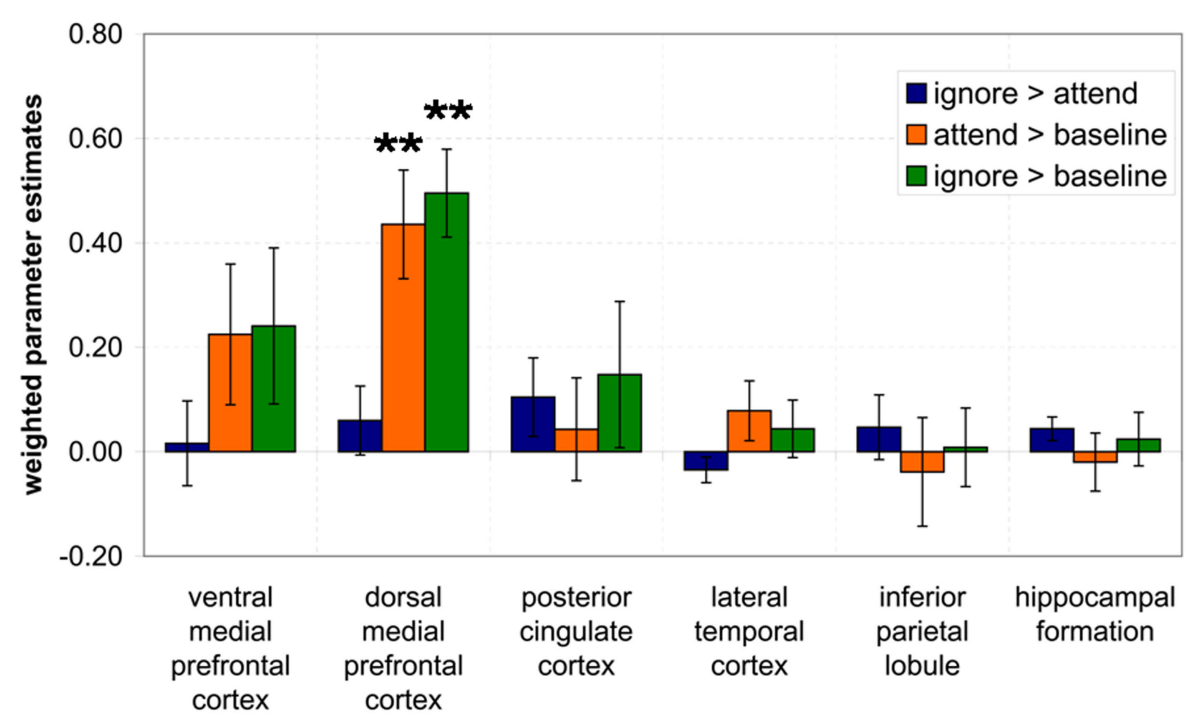

FIGURE 3 | Results of ROI analysis using typical DMN ROls. Weighted contrast parameter estimates were extracted from three contrasts ([ignore > attend], [attend $>$ baseline], [ignore $>$ baseline]) and entered into a one-sample $t$-test. ${ }^{*} p<0.008,{ }^{* *} p<0.001$. 


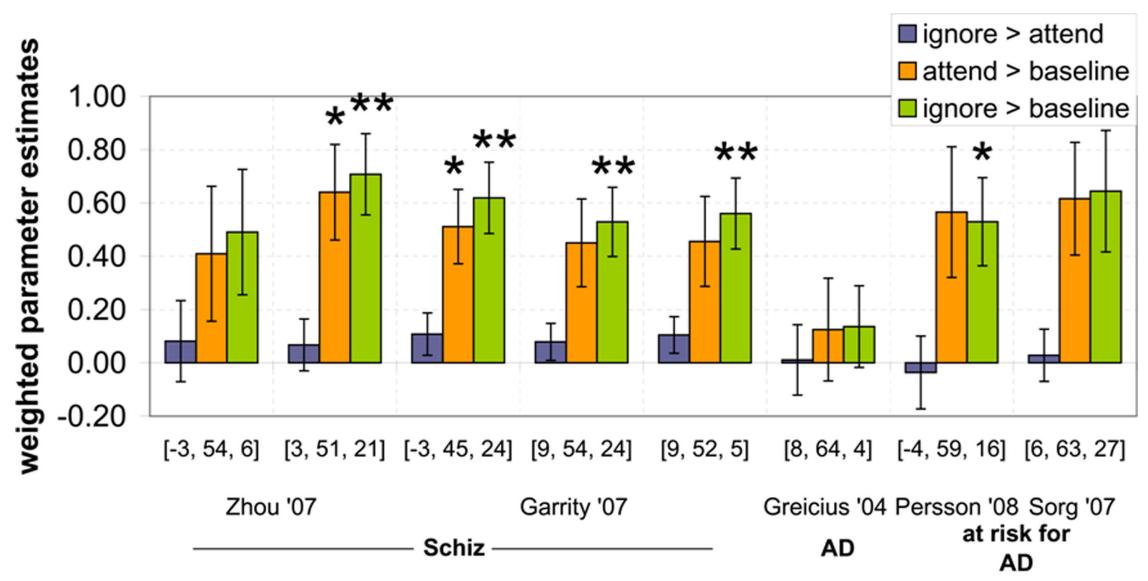

Functional ROls within dorsal medial prefrontal cortex

$[x, y, z]$ MNI coordinates

FIGURE 4 | Results of ROI analysis using clinical DMN ROIs. Weighted contrast parameter estimates were extracted from three contrasts ([ignore > attend], [attend $>$ baseline], [ignore $>$ baseline]) and entered into a one-sample $t$-test. ${ }^{*} p<0.006,{ }^{*} p<0.001$.

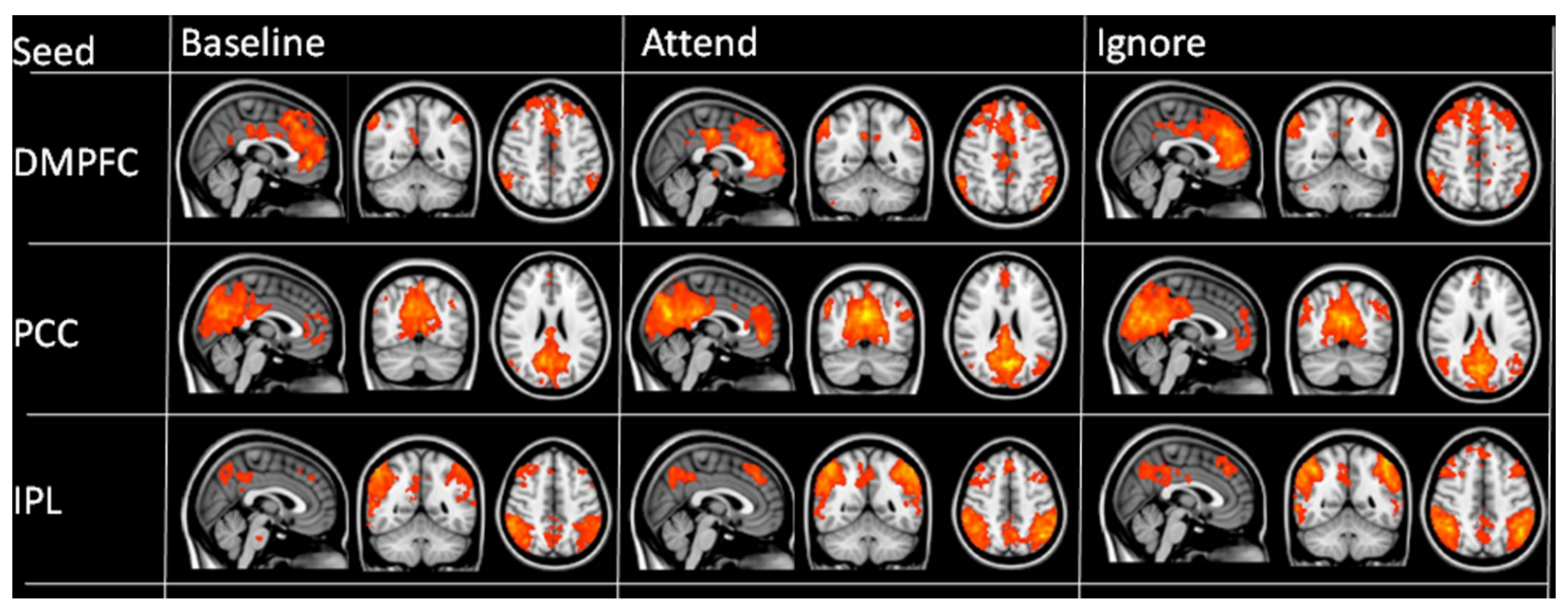

FIGURE 5 | Functional connectivity maps for each of the three conditions obtained using structural seeds as defined in text. Images thresholded at $p<0.005$ with a 20-voxel cluster threshold; color range from 2 (red) to $>5$ (yellow).

Analyses using the dmPFC ROI defined in line with Persson et al. (2008) yielded similar results (Figure 6). For the contrast ignore $>$ baseline, significantly greater connectivity was apparent in inferior parietal cortex bilaterally [left, $t(16)=5.23$; $p<0.01$; right, $t(16)=5.22 ; p<0.05$ ], as well as the left superior frontal gyrus $[t(16)=4.71 ; p<0.05]$. The contrast of attend $>$ baseline for the Persson ROI revealed a single cluster of significantly greater dmPFC connectivity during the "attend" condition in a region bordering the left inferior parietal cortex and posterior superior temporal gyrus $[t(16)=4.66 ; p<0.01]$.

\section{DISCUSSION}

Our results suggest that the dmPFC, a component of the DMN, is sensitive to different approaches to SBN during a wakeful, resting state in typical adults. The dmPFC exhibited increased activation when healthy participants attended to or ignored SBN compared to when they were not given specific instructions on how to rest (and how to attend to SBN). Other regions in the DMN (ventral medial prefrontal cortex, lateral temporal cortex, inferior parietal lobule, hippocampal formation, and posterior cingulate cortex) did not significantly differ with different rest period instructions in the direct comparisons using the GLM. None of the examined DMN regions (including dmPFC) exhibited significant differences when participants attended to SBN compared to when they ignored SBN. However, follow-up connectivity analyses revealed significant differences in the connectivity network within typical DMN regions (e.g., inferior parietal cortex) when comparing the three conditions. This was observed using seeds within $\mathrm{dmPFC}$ regions which were derived from previous studies that reported differences in the DMN between patients and healthy controls. 


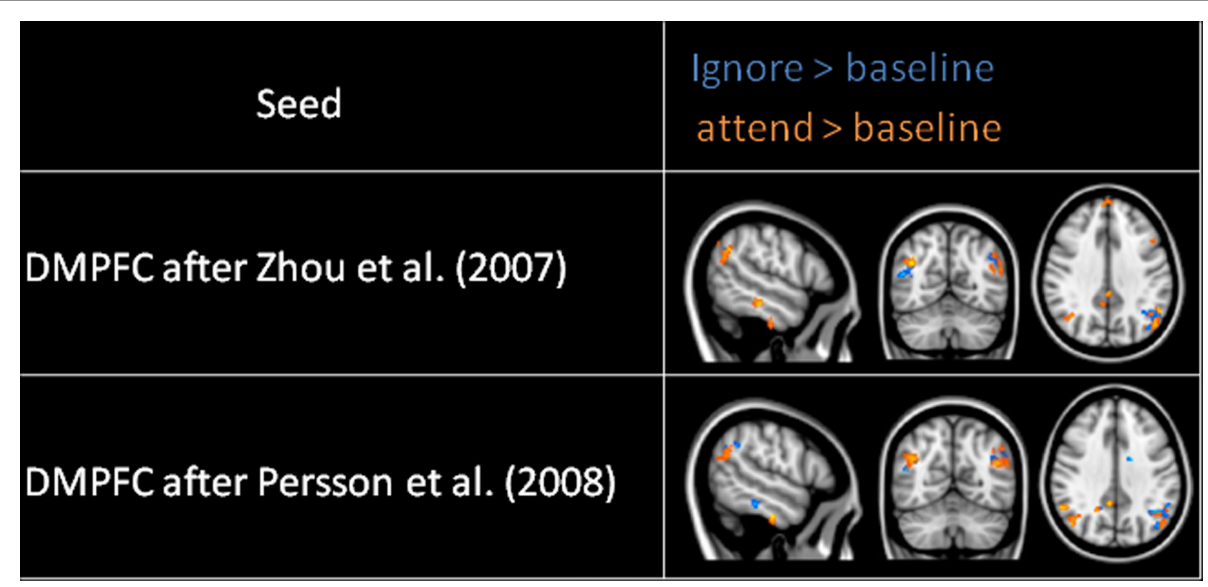

FIGURE 6 | Functional connectivity map obtained using dmPFC defined after Zhou et al. (2007; schizophrenia literature) and Persson et al. (2008;

Alzheimer's disease literature). Ignore $>$ baseline is represented in blue; attend $>$ baseline in orange. Image thresholded at $p<0.005,20$-voxel threshold, colors run from 2 (dark) to $>5$ (light).

The effects of specific resting instructions on dmPFC coincide with effects observed in previous studies on DMN alterations in patients with schizophrenia and Alzheimer's disease and individuals at risk for developing Alzheimer's disease. This suggests that some of the previously reported DMN differences in dmPFC in these populations may be partially influenced by different approaches to rest. This is particularly true if a patient group's pathology makes it more likely that they will systematically treat rest differently; for instance, in patients with auditory hallucinations. Unfortunately, unlike difference in the eye position during rest, differences in the attention toward SBN cannot be observed behaviorally. Our study cannot conclude that the previously observed differences in $\mathrm{dmPFC}$ between patients and healthy volunteers are effects of a systematic bias in the approach how to rest. However, our study shows that instructions on how to attend to SBN lead to alterations within previously reported components of the DMN, and therefore a careful interpretation of results in patient populations which may show a systematic bias are important.

For our sample of healthy adults, one possible explanation for increased dmPFC activation when ignoring or attending to SBN compared to baseline is an increased awareness of external stimuli. One of the hypothesized functions of the DMN is to monitor the external environment (Gusnard and Raichle, 2001). Consistent with this notion, activation in dmPFC was greater when participants were instructed to attend to or ignore one particular type of external stimuli: SBN. Whether the effort was to ignore or attend to SBN, the participant's awareness of SBN should be greater with instruction than with no instruction at all, which may explain the increased activation in dmPFC.

Our data suggest that the dmPFC, rather than the DMN as a complete network, is impacted by specific resting instructions with regard to SBN. Medial prefrontal areas have specifically been implicated in self-referential processing (Gusnard et al., 2001). The resting instructions used in the specific instruction phase of this experiment involve internal monitoring of one's own attentional resources, which may require an increase in self-awareness. If this is the case, then the self-referential processing during the specific instruction phase is beyond that required during the baseline phase. Interestingly, ventral medial prefrontal cortex was not significantly influenced by specific instructions for rest periods, although it is also implicated in self-referential processing.

With respect to DMN studies on clinical populations, our results raise the possibility that some of the previously reported differences may be confounded by different approaches to rest. These results are limited to the dmPFC rather than the DMN as a whole. However, it is possible that DMN differences between patients and controls in the $\mathrm{dmPFC}$ are at least in part explained by different approaches to rest in the presence of SBN. In the presence of SBN, patients with Alzheimer's disease or schizophrenia may employ different resting strategies than healthy adults. For instance, patients may actively attend to or ignore SBN when instructed to relax and stay still; this might cause increased dmPFC activation in the patient group only. Interestingly, DMN hyperactivity (including dmPFC) has been observed in several studies on patients with schizophrenia (Garrity et al., 2007; Zhou et al., 2007; Whitfield-Gabrieli et al., 2009), a disease hypothesized to be characterized by hypersensitivity to internal thoughts and the external environment (Broyd et al., 2009).

It is worth noting that of the five papers used to derive clinical ROIs, only one (Persson et al., 2008) used a block design, as was used in the current study. The other four studies used functional connectivity analyses to derive DMN ROIs. Our follow-up connectivity analyses also revealed differences in the connectivity network between the three conditions when the seed was placed in dmPFC regions derived from Zhou et al. (2007) and Persson et al. (2008). Differences were observed especially in the parietal-dmPFC connectivity for both the attend and the ignore conditions when compared to baseline. Interestingly, there have been several studies reporting alterations in the connectivity between frontal and parietal regions in patient groups compared to healthy controls (e.g., Woodward et al., 2009; in schizophrenia or Liao et al., 2010 for social anxiety disorder). Our results show that differences in the instructions on how to rest can alter the dmPFC-inferior parietal connectivity network. 
Our study is limited by a lack of behavioral data to verify that participants followed different resting instructions. Given the design of our experiment, however, collecting behavioral data would necessarily introduce a goal-directed task and would therefore undermine efforts to examine DMN activity in the absence of goal-directed activity. We used a data-driven approach to address this issue, including only participants with positive weighted parameter estimates in primary auditory regions (a bilateral anatomical ROI of Heschl's gyrus) for the attending to SBN compared to ignoring SBN contrast [attend > ignore]. Attention to auditory stimuli has previously been shown to modulate activity in primary auditory cortex (Jancke et al., 1999); in the study, increased attention correlated with increased activity in primary auditory cortex in healthy populations. Those individuals with greater activation in attend $>$ ignore contrast in primary auditory cortex were therefore most likely those who followed the given instructions. Thus, activation pattern in primary auditory cortex for attend > ignore was used as a proxy for behavioral data in the current study.

An additional limitation is that the baseline condition was collected in a single initial 160 s block, while the "attend" and "ignore" conditions were collected in alternating 20 s blocks (with the number of scans in each condition being equivalent). Our goal of comparing "baseline" rest to resting conditions in which subjects ignored or attended SBN required a baseline in which subjects were not made consciously aware of SBN; as such alternating all three conditions was unfeasible. We note that the dorsolateral PFC has previously been observed to respond differentially to unattended and attended stimuli (e.g., Walter et al., 2009). Our failure to observe such an effect may be a result of the temporal characteristics of the study design; however, the differences between these conditions and

\section{REFERENCES}

Amaro, E. Jr., Williams, S. C., Shergill, S. S., Fu, C. H., MacSweeney, M., Picchioni, M. M., Brammer, M. J., and McGuire, P. K. (2002). Acoustic noise and functional magnetic resonance imaging: current strategies and future prospects. J. Magn. Reson. Imaging 16, 497-510.

Bai, F., Zhang, Z., Yu, H., Shi, Y., Yuan, Y., Zhu, W., Zhang, X., and Qian, Y. (2008). Default-mode network activity distinguishes amnestic type mild cognitive impairment from healthy aging: a combined structural and resting-state functional MRI study. Neurosci. Lett. 438, 111-115.

Benjamini, Y., and Hochberg, Y. (1995). Controlling the false discovery rate: a practical and powerful approach to multiple testing. J. R. Stat. Soc. B Stat. Methodol. 57, 289-300.

Benjamini, Y., and Yekutieli, D. (2001). The control of the false discovery rate in multiple testing under dependency. Ann. Stat. 29, 1165-1188.

Binder, J. R., Frost, J. A., Hammeke, T. A., Bellgowan, P. S., Rao, S. M., and Cox, R. W. (1999). Conceptual processing

the resting baseline remain unchanged. A logical follow-up study should examine the suggested effect in three blocks with equal duration (more than 5 min each).

As previously suggested for the eye position (eyes open or eyes closed), our findings suggest that the DMN in dmPFC may be subject to confounds from different approaches to rest in the presence of SBN (Morcom and Fletcher, 2007; Raichle and Snyder, 2007; McAvoy et al., 2008). Papers examining the DMN often have non-specific, variable resting instructions; rest period instructions should therefore be carefully considered when developing controlled MR experiments on the DMN, along with varying levels of SBN (Gaab et al., 2007). This issue may be avoided by providing research participants with consistent instructions for rest periods and/or employing MR scanning protocols that minimize SBN. Our findings have specific relevance to DMN studies on clinical populations (as shown by our clinical ROI analyses) but are also relevant to studies examining typical populations. Variables such as experience in the MR scanner environment (e.g., a first-time research participant versus a seasoned researcher as a member of the control group) may contribute to different resting strategies as well. Child research participants may also use different resting strategies because of heightened anxiety or unfamiliarity with the MR scanning environment (Raschle et al., 2009).

Further studies on the sensitivity of the DMN to such factors is essential to understanding the nature of the DMN and how we may use it to characterize various neurological and psychiatric disorders.

\section{ACKNOWLEDGMENT}

We thank Sheeba Arnold for her excellent help with the connectivity analyses.

an independent component analysis. J. Neurosci. 26, 10222-10231.

A fung the conscious resting state. A functional MRI study. J. Cogn. Neurosci. 11, 80-95.

Bluhm, R. L., Miller, J., Lanius, R. A., Osuch, E. A., Boksman, K., Neufeld, R. W., Theberge, J., Schaefer, B., and Williamson, P. (2007). Spontaneous low-frequency fluctuations in the BOLD signal in schizophrenic patients: anomalies in the default network. Schizophr. Bull. 33, 1004-1012.

Broyd, S. J., Demanuele, C., Debener, S., Helps, S. K., James, C. J., and SonugaBarke, E. J. (2009). Default-mode brain dysfunction in mental disorders: a systematic review. Neurosci. Biobehav. Rev. 33, 279-296.

Buckner, R. L., Andrews-Hanna, J. R., and Schacter, D. L. (2008). The brain's default network: anatomy, function, and relevance to disease. Ann. N. Y. Acad. Sci. 1124, 1-38.

Celone, K. A., Calhoun, V. D., Dickerson, B. C., Atri, A., Chua, E. F., Miller, S. L., DePeau, K., Rentz, D. M., Selkoe, D. J., Blacker, D., Albert, M. S., and Sperling, R. A. (2006). Alterations in memory networks in mild cognitive impairment and Alzheimer's disease:
Firbank, M. J., Blamire, A. M., Krishnan, M. S., Teodorczuk, A., English, P., Gholkar,A., Harrison, R., and O'Brien, J. T. (2007). Atrophy is associated with posterior cingulate white matter disruption in dementia with Lewy bodies and Alzheimer's disease. Neuroimage 36, 1-7.

Fox, M. D., and Raichle, M. E. (2007) Spontaneous fluctuations in brain activity observed with functional magnetic resonance imaging. Nat. Rev. Neurosci. 8, 700-711.

Fransson, P. (2006). How default is the default mode of brain function? Further evidence from intrinsic BOLD signal fluctuations. Neuropsychologia 44, 2836-2845.

Friston, K. J., Holmes, A. P., Poline, J. B., Grasby, P. J., Williams, S. C., Frackowiak, R. S., and Turner, R. (1995). Analysis of fMRI time-series revisited. Neuroimage 2, 4553.

Gaab, N., Gabrieli, J. D., and Glover, G. H. (2007). Assessing the influence of scanner background noise on auditory processing. II. An fMRI study comparing auditory processing in the absence and presence of recorded scanner noise using a sparse design. Hum. Brain Mapp. 28, 721-732.

Gaab, N., Gabrieli, J. D., and Glover, G. H. (2008). Resting in peace or noise: scanner background noise suppresses default-mode network. Hum. Brain Mapp. 29, 858-867.

Garrity, A. G., Pearlson, G. D., McKiernan, K., Lloyd, D., Kiehl, K. A., and Calhoun, V.D. (2007). Aberrant "default mode" functional connectivity in schizophrenia. Am. J. Psychiatry 164, 450-457.

Greicius, M. D., Flores, B. H., Menon, V., Glover, G. H., Solvason, H. B., Kenna, H., Reiss, A. L., and Schatzberg, A. F. (2007). Resting-state functional connectivity in major depression: abnormally increased contributions from subgenual cingulate cortex and thalamus. Biol. Psychiatry 62, 429-437.

Greicius, M. D., Krasnow, B., Reiss, A. L., and Menon, V. (2003). Functional connectivity in the resting brain: a network analysis of the default mode hypothesis. Proc. Natl. Acad. Sci. U.S.A. 100, 253-258. 
Greicius, M. D., Srivastava, G., Reiss, A. L., and Menon, V. (2004). Defaultmode network activity distinguishes Alzheimer's disease from healthy aging: evidence from functional MRI. Proc. Natl. Acad. Sci. U.S.A. 101, 4637-4642.

Gusnard, D.A., Akbudak, E., Shulman, G. L., and Raichle, M. E. (2001). Medial prefrontal cortex and self-referential mental activity: relation to a default mode of brain function. Proc. Natl. Acad. Sci. U.S.A. 98, 4259-4264.

Gusnard, D. A., and Raichle, M.E. (2001). Searching for a baseline: functional imaging and the resting human brain. Nat. Rev. Neurosci. 2, 685-694.

Harrison, B. J., Yucel, M., Pujol, J., and Pantelis, C. (2007). Task-induced deactivation of midline cortical regions in schizophrenia assessed with fMRI. Schizophr. Res. 91, 82-86.

He, Y., Wang, L., Zang, Y., Tian, L. Zhang, X., Li, K., and Jiang, T. (2007). Regional coherence changes in the early stages of Alzheimer's disease: a combined structural and resting-state functional MRI study. Neuroimage 35, 488-500.

Jancke, L., Mirzazade, S., and Shah, N. J. (1999). Attention modulates activity in the primary and the secondary auditory cortex: a functional magnetic resonance imaging study in human subjects. Neurosci. Lett. 266, 125-128.

Kennedy, D. P., and Courchesne, E. (2008). Functional abnormalities of the default network during self- and other-reflection in autism. Soc. Cogn. Affect. Neurosci. 3, 177-190.

Kennedy,D.P., Redcay, E., and Courchesne, E. (2006). Failing to deactivate: resting functional abnormalities in autism. Proc. Natl. Acad. Sci. U.S.A. 103, 8275-8280.

Liao, W., Chen, H., Feng, Y., Mantini, D., Gentili, C., Pan, Z., Ding, J., Duan, X., Qiu, C., Lui, S., Gong, Q., and Zhang, W. (2010). Selective aberrant functional connectivity of resting state networks in social anxiety disorder. Neuroimage 52, 1549-1558.

Liu, Y., Wang, K., Yu, C., He, Y., Zhou, Y., Liang, M., Wang, L., and Jiang, T. (2008). Regional homogeneity, functional connectivity and imaging markers of Alzheimer's disease: a review of resting-state fMRI studies. Neuropsychologia 46, 1648-1656.

Lustig, C., Snyder, A. Z., Bhakta, M., O’Brien, K. C., McAvoy, M., Raichle, M. E., Morris, J. C., and Buckner, R. L. (2003). Functional deactivations: change with age and dementia of the Alzheimer type. Proc. Natl. Acad. Sci. U.S.A. 100, 14504-14509.

Maldjian,J.A.,Laurienti,P.J., and Burdette,J. H. (2004). Precentral gyrus discrepancy in electronic versions of the Talairach atlas. Neuroimage 21, 450-455.
Maldjian, J. A., Laurienti, P. J., Kraft, R. A., and Burdette, J. H. (2003). An automated method for neuroanatomic and cytoarchitectonic atlasbased interrogation of fMRI data sets. Neuroimage 19, 1233-1239.

Mason, M. F., Norton, M. I., Van Horn, J. D., Wegner, D. M., Grafton, S. T., and Macrae, C. N. (2007). Wandering minds: the default network and stimulus-independent thought. Science 315, 393-395.

Mazoyer, B., Zago, L., Mellet, E., Bricogne, S., Etard, O., Houde, O., Crivello, F., Joliot, M., Petit, L., and TzourioMazoyer,N. (2001). Cortical networks for working memory and executive functions sustain the conscious resting state in man. Brain Res. Bull. 54, 287-298.

McAvoy, M., Larson-Prior, L., Nolan, T., S., Vaishnavi, S. N., Raichle, M.E., and d'Avossa, G. (2008). Resting states affect spontaneous BOLD oscillations in sensory and paralimbic cortex. $J$. Neurophysiol. 100, 922-931.

Morcom, A. M., and Fletcher, P. C. (2007) Does the brain have a baseline? Why we should be resisting a rest. Neuroimage 37, 1073-1082.

Novitski, N., Alho, K., Korzyukov, O., Carlson, S., Martinkauppi, S., Escera, C., Rinne, T., Aronen, H. J., and Näätänen, R. (2001). Effects of acoustic gradient noise from functional magnetic resonance imaging on auditory processing as reflected by event-related brain potentials. Neuroimage 14(Pt 1), 244-251.

Persson, J., Lind, J., Larsson, A., Ingvar, M., Sleegers, K., Van Broeckhoven, C., Adolfsson, R., Nilsson, L. G., and Nyberg, L. (2008). Altered deactivation in individuals with genetic risk for Alzheimer's disease. Neuropsychologia 46, 1679-1687.

Pomarol-Clotet, E., Salvador, R., Sarro, S., Gomar, J., Vila, F., Martinez, A., Guerrero, A., Ortiz-Gil, J., Sans-Sansa, B., Capdevila, A., Cebamanos, J. M., and McKenna, P. J. (2008). Failure to deactivate in the prefrontal cortex in schizophrenia: dysfunction of the default mode network? Psychol. Med. 38, 1185-1193.

Price, D. L., De Wilde, J. P., Papadaki, A. M., Curran, J. S., and Kitney, R. I. (2001). Investigation of acoustic noise on $15 \mathrm{MRI}$ scanners from $0.2 \mathrm{~T}$ to $3 \mathrm{~T}$. J. Magn. Reson. Imaging 13, 288-293.

Raichle, M. E., MacLeod, A. M., Snyder, A. Z., Powers, W. J., Gusnard, D. A., and Shulman, G. L. (2001). A default mode of brain function. Proc. Natl. Acad. Sci. U.S.A. 98, 676-682.

Raichle, M. E., and Snyder, A. Z. (2007). A default mode of brain function: a brief history of an evolving idea.
Neuroimage 37, 1083-1090; discussion 1097-1099.

Raschle, N. M., Lee, M., Buechler, R., Christodoulou, J. A., Chang, M., and Vakil,M. (2009). Making MR imaging child's play - pediatric neuroimaging protocol, guidelines and procedure. $J$. Vis. Exp. 29.

Ravicz, M.E., Melcher, J. R., and Kiang, N. Y. (2000). Acoustic noise during functional magnetic resonance imaging. $J$. Acoust. Soc. Am. 108, 1683-1696.

Rombouts, S. A., Barkhof, F., Goekoop, R. Stam, C. J., and Scheltens, P. (2005). Altered resting state networks in mild cognitive impairment and mild Alzheimer's disease: an fMRI study. Hum. Brain Mapp. 26, 231-239.

Sauer, J., ffytche, D. H., Ballard, C., Brown, R. G., and Howard, R. (2006). Differences between Alzheimer's disease and dementia with Lewy bodies: an fMRI study of task-related brain activity. Brain 129(Pt 7), 1780-1788.

Shulman, G. L., Fiez, J. A., Corbetta, M., Buckner, R. L., Miezin, F. M. Raichle, M. E., and Petersen, S. (1997). Common blood flow changes across visual tasks: II. Decreases in cerebral cortex. J. Cogn. Neurosci. 9, 648-663.

Sorg, C., Riedl, V., Muhlau, M., Calhoun, V. D., Eichele, T., Laer, L., Drzezga, A., Forstl, H., Kurz, A., Zimmer, C., and Wohlschlager, A. M. (2007). Selective changes of resting-state networks in individuals at risk for Alzheimer's disease. Proc. Natl. Acad. Sci. U.S.A. 104 18760-18765.

Talavage, T.M., and Edmister,W.B. (2004). Nonlinearity of FMRI responses in human auditory cortex. Hum. Brain Mapp. 22, 216-228.

Tian, L., Jiang, T., Wang, Y., Zang, Y., He, Y., Liang, M., Sui, M., Cao, Q., Hu, S., Peng, M., and Zhuo, Y. (2006). Altered resting-state functional connectivity patterns of anterior cingulate cortex in adolescents with attention deficit hyperactivity disorder. Neurosci. Lett. 400, 39-43.

Tomasi, D., Caparelli, E. C., Chang, L. and Ernst, T. (2005). fMRI-acoustic noise alters brain activation during working memory tasks. Neuroimage 27, 377-386.

Tzourio-Mazoyer, N., Landeau, B. Papathanassiou, D., Crivello, F., Etard, O., Delcroix, N., Mazoyer, B., and Joliot, M. (2002). Automated anatomical labeling of activations in SPM using a macroscopic anatomical parcellation of the MNI MRI single-subject brain. Neuroimage 15, 273-289.

Walter, M., Matthiä, C., Wiebking, C., Rotte, M., Tempelmann, C., Bogerts, B., Heinze, H. J., and Northoff, G (2009). Preceding attention and the dorsomedial prefrontal cortex: process specificity versus domain dependence. Hum. Brain Mapp. 30, 312-326.

Wang, L., Zang, Y., He, Y., Liang, M., Zhang, X., Tian, L., Wu, T., Jiang, T., and Li, K. (2006). Changes in hippocampal connectivity in the early stages of Alzheimer's disease: evidence from resting state fMRI. Neuroimage 31, 496-504.

Wermke, M., Sorg, C., Wohlschlager, A. M., and Drzezga, A. (2008). A new integrative model of cerebral activation, deactivation and default mode function in Alzheimer's disease. Eur. J. Nucl. Med. Mol. Imaging 35(Suppl. 1), S12-S24.

Whitfield-Gabrieli, S., Thermenos, H.W., Milanovic, S., Tsuang, M. T., Faraone, S. V., McCarley, R. W., Shenton, M. E., Green, A. I., Nieto-Castanon, A., LaViolette, P., Wojcik, J., Gabrieli, J. D., and Seidman, L. J. (2009). Hyperactivity and hyperconnectivity of the default network in schizophrenia and in first-degree relatives of persons with schizophrenia. Proc. Natl. Acad. Sci. U.S.A. 106, 1279-1284.

Woodward, N. D., Waldie, B., Rogers, B., Tibbo, P., Seres, P., and Purdon, S. E. (2009). Abnormal prefrontal cortical activity and connectivity during response selection in first episode psychosis, chronic schizophrenia, and unaffected siblings of individuals with schizophrenia. Schizophr. Res. 109, 182-190

Zhou, Y., Liang, M., Tian, L., Wang, K., Hao, Y., Liu, H., Liu, Z., and Jiang, T. (2007). Functional disintegration in paranoid schizophrenia using resting-state fMRI. Schizophr. Res. 97, 194-205.

Conflict of Interest Statement: The authors declare that the research was conducted in the absence of any commercial or financial relationships that could be construed as a potential conflict of interest.

Received: 17 February 2010; accepted: 29 October 2010; published online: 01 December 2010.

Citation: Benjamin C, Lieberman DA, Chang M, Ofen N, Whitfield-Gabrieli S, GabrieliJDE and Gaab N(2010) The influence of rest period instructions on the default mode network. Front. Hum. Neurosci. 4:218. doi: 10.3389/fnhum.2010.00218 Copyright (ㄷ) 2010 Benjamin, Lieberman, Chang, Ofen, Whitfield-Gabrieli, Gabrieli and Gaab. This is an open-access article subject to an exclusive license agreement between the authors and the Frontiers Research Foundation, which permits unrestricted use, distribution, and reproduction in any medium, provided the original authors and source are credited. 